\title{
Sarcopenia Is an Independent Risk Factor for NAFLD in COPD: A Nationwide Survey (KNHANES 2008-20II)
}

This article was published in the following Dove Press journal: International Journal of Chronic Obstructive Pulmonary Disease

\author{
Kyung Soo Hong (D' \\ Min Cheol Kim iD ${ }^{2}$ \\ June Hong Ahn (D) \\ 'Division of Pulmonary and Allergy, \\ Department of Internal Medicine, College \\ of Medicine, Yeungnam University and \\ Regional Center for Respiratory \\ Diseases, Yeungnam University Medical \\ Center, Daegu, South Korea; ${ }^{2}$ Division of \\ Gastroenterology, Department of \\ Internal Medicine, Yeungnam University \\ Medical Center, College of Medicine, \\ Yeungnam University, Daegu, South \\ Korea
}

Background: Nonalcoholic fatty liver disease (NAFLD) is highly prevalent in patients with chronic obstructive pulmonary disease (COPD) and is independently associated with cardiometabolic comorbidities and systemic inflammation. Although several factors are associated with NAFLD, the influence of sarcopenia on NAFLD has not been fully determined in patients with COPD. We explored whether sarcopenia is associated with NAFLD in a COPD population.

Methods: Data from the Korean National Health and Nutrition Examination Surveys 2008-2011 were analyzed. The subjects were defined as having NAFLD when they had a hepatic steatosis index (HSI) score $>36$, which is a previously validated NAFLD prediction score. Sarcopenia_BMI (=total appendicular skeletal muscle mass $[\mathrm{kg}] /$ body mass index $\left[\mathrm{kg} / \mathrm{m}^{2}\right]$ ), sarcopenia_BW (=total appendicular skeletal muscle mass $[\mathrm{kg}]$ /body weight $[\mathrm{kg}] \times 100)$, and sarcopenia_height $(=$ total appendicular skeletal muscle mass $(\mathrm{kg}) /$ height $^{2}(\mathrm{~m})$ ) measured using dual-energy X-ray absorptiometry was used to diagnose sarcopenia.

Results: NAFLD was identified in $124(14.6 \%)$ of 850 COPD subjects using the HSI. Multivariable logistic analyses adjusted for age, sex, hypertension, diabetes mellitus (DM), forced vital capacity (FVC), and metabolic syndrome demonstrated that sarcopenia (sarcopenia_BMI, odds ratio $[\mathrm{OR}]=1.95 ; 95 \%$ confidence interval $[\mathrm{CI}], 1.11-3.46, \mathrm{p}=$ 0.022 ; sarcopenia_BW, OR $=2.25 ; 95 \% \mathrm{CI}, 1.30-3.92, \mathrm{p}=0.004)$ was associated with NAFLD in patients with COPD. The proportion of sarcopenia BMI was higher in patients with a high fibrotic burden from NAFLD (Q3, Q4) than in subjects with a low fibrotic burden from NALFD (Q1, Q2) $(54.8 \%$ vs $24.2 \%, \mathrm{p}=0.024)$. The proportion of sarcopenia_BW was also higher in patients with a high fibrotic burden from NAFLD than in patients with a low fibrotic burden from NAFLD $(51.6 \%$ vs $30.6 \%, \mathrm{p}=0.029)$.

Conclusion: Sarcopenia was associated with an increased risk for NAFLD in patients with COPD, independent of age, sex, lung function, and metabolic factors. Sarcopenic COPD was also associated with a high fibrotic burden in NAFLD patients. Pulmonologists should be aware of possible liver comorbidities in the sarcopenic COPD phenotype.

Keywords: COPD, sarcopenia, NAFLD

\section{Introduction}

Nonalcoholic fatty liver disease (NAFLD) is hepatic fat accumulation with evidence of hepatic steatosis, either by imaging or by histology; it excludes secondary causes, such as heavy alcohol consumption, chronic viral hepatitis, use of steatogenic medication, and a hereditary disorder. The long term prognosis of NAFLD patients includes increased overall mortality, increased frequency of cardiovascular
Division of Pulmonary and Allergy,

Department of Internal Medicine, College of Medicine, Yeungnam University and Regional Center for Respiratory Diseases, Yeungnam University Medical Center, 170 Hyeonchung-ro, Nam-gu, Daegu 424I5, South Korea

Email fireajh@gmail.com 
disease, and increased liver-related mortality. ${ }^{1}$ NAFLD presents a wide spectrum of liver damage, ranging from fatty liver to steatohepatitis and cirrhosis. ${ }^{2}$ Several studies have reported an association between chronic obstructive pulmonary disease (COPD) and NAFLD. Liver disease is highly prevalent in patients with COPD, and NAFLD is associated with poor pulmonary function, particularly a restrictive ventilatory defect. ${ }^{3-7}$ Patients with NAFLD and liver fibrosis have more cardiovascular events and a higher mortality from COPD. ${ }^{8}$

Sarcopenia frequently occurs in patients with COPD, with a prevalence of $15-25 \%$ in previous studies. Sarcopenia has important clinical meaning in patients with COPD, such as reduced physical activity, reduced exercise tolerance, reduced quality of life, and reduced survival. ${ }^{9}$

Several studies have reported the relationship between sarcopenia and NAFLD in the general population. Koo et al reported that low muscle mass is associated with an increased risk for NAFLD and significant fibrosis using a large biopsyproven NAFLD cohort. ${ }^{10}$ Lee et al also reported that sarcopenia is associated with an increased risk for NAFLD, and sarcopenia is associated with significant liver fibrosis in NAFLD patients according to nationwide surveys. ${ }^{11,12}$ Sarcopenia and NAFLD share common pathophysiological mechanisms, such as insulin resistance, vitamin D deficiency, and chronic low-grade inflammation, which are also common mechanisms of COPD. ${ }^{13}$ However, no study has assessed the relationship between sarcopenia and NAFLD in a COPD cohort until now. We investigated the relationship between sarcopenia and NAFLD in patients with COPD, using a nationwide cross-sectional survey database (The Korea National Health and Nutrition Examination Survey, KNHANES).

\section{Methods}

\section{Study Subjects}

The KNHANES is a national cross-sectional survey system that has evaluated the health and nutrition status of Koreans since 1998 and is conducted by the Korean Centers for Disease Control and Prevention (KCDC). The survey uses a stratified, multi-stage clustered probability design to sample non-institutionalized Korean citizens living in Korea. The KNHANES consists of three component surveys: a health interview, a health examination and a nutrition survey. ${ }^{14,15}$

A flow diagram of the study subjects is shown in Figure 1. Of the 21,303 subjects from the KNHANES 2008-2011, we initially selected 12,730 subjects aged $\geq 40$ years. Subjects with missing data for dual-energy X-ray absorptiometry (DXA), body mass index (BMI), platelets, aspartate transaminase (AST), or alanine transaminase (ALT) were excluded $(n=2727)$. Subjects who did not meet the criteria for NAFLD were also excluded ( $\mathrm{n}=$ 2819), as follows: positive serological markers for hepatitis $\mathrm{B}$ or positive serological markers for hepatitis $\mathrm{C}$ or excessive alcohol consumption $(>210 \mathrm{~g}$ alcohol per week

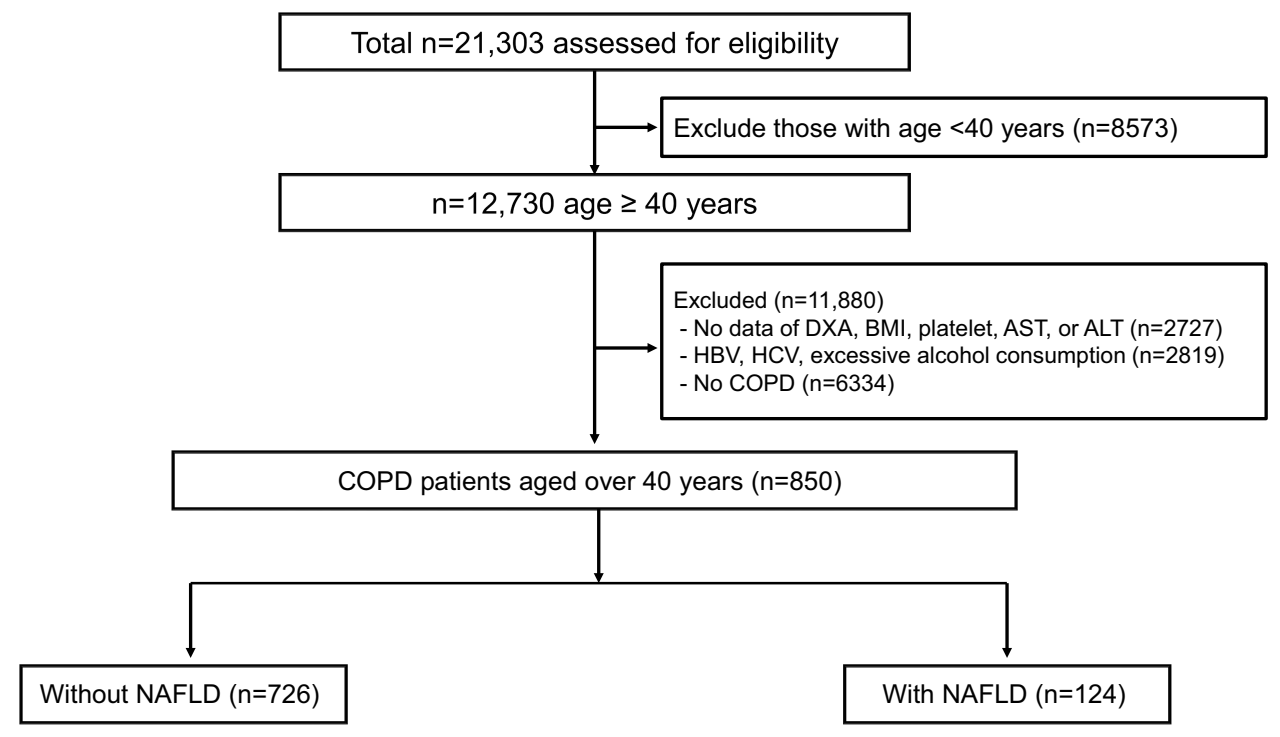

Figure I A flow diagram of the study subjects in the KNHANES IV and V (2008-20II).

Abbreviations: ALT, alanine aminotransferase; AST, aspartate aminotransferase; BMI, body mass index; COPD, chronic obstructive pulmonary disease; DXA, dual-energy X-ray absorptiometry; HBV, hepatitis B virus; HCV, hepatitis C virus; NAFLD, nonalcoholic fatty liver disease. 
for men and $>140 \mathrm{~g}$ alcohol per week for women). A total of 6334 subjects without COPD were further excluded. Ultimately, 850 subjects were included in the analyses and categorized according to the presence of NAFLD.

\section{Clinical and Laboratory Measurements}

Trained interviewers administered a standardized questionnaire to the study subjects to determine their age, height, body weight, waist circumference, and smoking status. Blood samples were collected after at least $8 \mathrm{~h}$ of fasting. All blood samples were processed, transported to a central testing center (Neodin Medical Institute, Seoul, Korea), and analyzed within $24 \mathrm{~h}$ after transport. The blood samples were analyzed using the KNHANES protocol and a Hitachi 7600 automated chemistry analyzer (Hitachi, Tokyo, Japan). Qualified technicians performed the spirometry, and these data were sent to the principal investigator to assess whether the results met the criteria for acceptability and reproducibility. Body composition was measured using DXA (QRA 4500A; Hologic Inc., Bedford, MA, USA).

\section{Definitions}

COPD was defined if forced expiratory volume in $1 \mathrm{~s}\left(\mathrm{FEV}_{1}\right)$ /forced vital capacity (FVC) was $<0.7$ by spirometry. NAFLD was defined according to a hepatic steatosis index $(\mathrm{HSI})>36$, which is a previously validated fatty liver prediction model: ${ }^{16}$

$\mathrm{HSI}=8 \times($ ALT/AST ratio $)+\mathrm{BMI}(+2$ for diabetes mellitus; +2 for female)

NAFLD severity with significant liver fibrosis was assessed using the NAFLD fibrosis score (NFS). ${ }^{17}$ Serum levels of albumin were not used because they were not included in the KNHANES data. Fibrosis severity was divided into quartiles according to the NFS.

Metabolic syndrome was defined according to the revised National Cholesterol Education Program Adult Treatment Panel III criteria, and the International Diabetes Foundation considering ethnic specific thresholds in waist circumference. ${ }^{18}$ Metabolic syndrome was diagnosed as the presence of three of five factors: waist circumference $\geq 90 \mathrm{~cm}$ in men and $\geq 80 \mathrm{~cm}$ in women; triglycerides $\geq 150 \mathrm{mg} / \mathrm{dL}$ or taking medication; high-density lipoprotein (HDL) cholesterol $\leq 40 \mathrm{mg} / \mathrm{dL}$ in men or $<50 \mathrm{mg} / \mathrm{dL}$ in women or taking medications; systolic blood pressure $\geq 130 \mathrm{mmHg}$ or diastolic blood pressure $\geq 85 \mathrm{mmHg}$ or taking medications; and glucose $\geq 100 \mathrm{mg} / \mathrm{dL}$ or taking medications.

Appendicular skeletal muscle mass (ASM) was measured using DXA, as described above. We adopted three definitions of sarcopenia:
Sarcopenia_BMI $=$ total appendicular skeletal muscle mass $(\mathrm{kg}) /$ body mass index $\left(\mathrm{kg} / \mathrm{m}^{2}\right)$ was defined as ASM/ $\mathrm{BMI}<0.789$ in men or ASM/BMI $<0.512$ in women. The Foundation for the National Institutes of Health sarcopenia project recommends the ASM/BMI ratio. ${ }^{19}$

Sarcopenia_BW = total appendicular skeletal muscle mass $(\mathrm{kg}) /$ body weight $(\mathrm{kg}) \times 100$ was defined as less than two standard deviations (SDs) below the sex-specific mean for healthy young adults according to the 2008-2011 KNHANES data $(\mathrm{ASM} / \mathrm{BW} \times 100<29.0$ in men or ASM/ $\mathrm{BW} \times 100<22.9$ in women was defined as sarcopenia). ${ }^{10,20}$

Sarcopenia_height $=$ total appendicular skeletal muscle mass $(\mathrm{kg}) /$ height $^{2}(\mathrm{~m})$ was defined as ASM $/$ height $^{2}<$ $7.0 \mathrm{~kg} / \mathrm{m}^{2}$ in men or ASM $/$ height $^{2}<5.4 \mathrm{mg} / \mathrm{m}^{2}$ in women. Asian Working Group for Sarcopenia (AWGS) recommends using height-adjusted skeletal muscle mass. ${ }^{21}$

\section{Statistical Analyses}

As the KNHANES data are generated from a complex sampling survey design, all analyses were conducted using survey weighting. Continuous variables are expressed as mean \pm standard error and were compared to Student's $t$-test. Categorical variables were compared using the chi-square test. Multivariable logistic regression analyses were used to identify the independent association between sarcopenia and NAFLD in COPD subjects. Model 1 was adjusted for age and sex; model 2 was adjusted for model 1 and FVC; model 3 was adjusted for model 2 and hypertension and diabetes mellitus; and model 4 was adjusted for model 3 and metabolic syndrome.

All statistical analyses were performed using $\mathrm{R}$ statistical software (version 3.4.4, $\mathrm{R}$ Foundation for Statistical Computing, Vienna, Austria). A p-value $<0.05$ was considered significant.

\section{Ethics Statement}

This study was conducted in accordance with the Declaration of Helsinki. This study was approved by the Institutional Review Board of the KCDC (2008-04EXP-01-C, 200901CON-03-2C, 2010-02CON-21-C, 2011-02CON-06-C). Written informed consent was obtained from all patients.

\section{Results}

\section{Baseline Characteristics}

In total, 850 COPD patients were eligible for the study: 726 without NAFLD (85.4\%) and 124 with NAFLD (14.6\%). The baseline characteristics of the patients are presented in 
Table 1. The NAFLD subjects were younger $(65.4 \pm 9.5$ vs $63.0 \pm 10.2$ years, $\mathrm{p}=0.010)$, had a higher BMI $(22.9 \pm 2.4$ vs $\left.27.0 \pm 2.6 \mathrm{~kg} / \mathrm{m}^{2}, \mathrm{p}<0.001\right)$, and had a higher waist circumference $(82.5 \pm 7.8$ vs $93.6 \pm 7.3 \mathrm{~cm}, \mathrm{p}<0.001)$ than subjects without NAFLD. Subjects with NAFLD had a significantly lower ASM/BMI ratio $(0.8 \pm 0.2$ vs $0.7 \pm$ $0.2, \mathrm{p}<0.001)$ and ASM/BW ratio $(29.5 \pm 4.0 \mathrm{vs} 27.3 \pm 3.8$, $\mathrm{p}<0.001)$ than those without NAFLD. NAFLD subjects were significantly more likely to have comorbidities, such as metabolic syndrome ( $30.9 \%$ vs $68.5 \%, \mathrm{p}<0.001)$, hypertension $(47.1 \%$ vs $57.3 \%, \mathrm{p}<0.001)$, and diabetes mellitus $(13.1 \%$ vs $39.5 \%, \mathrm{p}<0.001)$ than subjects without NAFLD. The subjects with NAFLD showed a decrease in FVC compared to subjects without NAFLD $(89.7 \pm 15.5$ vs $85.3 \pm 15.3, \mathrm{p}=0.004)$. NAFLD subjects also had higher blood pressure, lower HDL cholesterol levels, higher triglyceride levels, higher liver enzyme levels, and higher glucose levels than those without NAFLD.

Table I Characteristics of COPD Patients According to NAFLD Status

\begin{tabular}{|c|c|c|c|c|}
\hline Variables & All $(n=850)$ & No NAFLD $(n=726,85.4 \%)$ & NAFLD $(n=124,14.6 \%)$ & P-value \\
\hline Age (years) & $65.0 \pm 9.6$ & $65.4 \pm 9.5$ & $63.0 \pm 10.2$ & 0.010 \\
\hline Male sex & $581(68.4)$ & $498(68.6)$ & $83(66.9)$ & 0.713 \\
\hline BMI $\left(\mathrm{kg} / \mathrm{m}^{2}\right)$ & $23.5 \pm 2.8$ & $22.9 \pm 2.4$ & $27.0 \pm 2.6$ & $<0.001$ \\
\hline Waist circumference $(\mathrm{cm})$ & $84.1 \pm 8.8$ & $82.5 \pm 7.8$ & $93.6 \pm 7.3$ & $<0.001$ \\
\hline Smoking status & & & & 0.485 \\
\hline Never smoker & $435(5 \mid .2)$ & $37 \mid(5 I . I)$ & $64(5 \mathrm{I} .6)$ & \\
\hline Ex-smoker & I78 (20.9) & I $48(20.4)$ & $30(24.2)$ & \\
\hline Current smoker & $237(27.9)$ & $207(28.5)$ & $30(24.2)$ & \\
\hline ASM (kg) & $18.2 \pm 4.0$ & $17.8 \pm 3.9$ & $19.6 \pm 4.4$ & $<0.001$ \\
\hline ASM/BMI $\left(m^{2}\right)$ & $0.8 \pm 0.2$ & $0.8 \pm 0.2$ & $0.7 \pm 0.2$ & $<0.001$ \\
\hline Sarcopenia_BMI & $174(20.5)$ & $|3|(\mid 8.0)$ & $43(34.7)$ & $<0.001$ \\
\hline ASM/BW (\%) & $29.2 \pm 4.0$ & $29.5 \pm 4.0$ & $27.3 \pm 3.8$ & $<0.001$ \\
\hline Sarcopenia_BW & $|8|(2 \mid .3)$ & $130(17.9)$ & $5 I(4 I .1)$ & $<0.001$ \\
\hline ASM/height ${ }^{2}$ & $6.8 \pm 1.0$ & $6.7 \pm 1.0$ & $7.3 \pm 1.0$ & $<0.001$ \\
\hline Sarcopenia_height & $278(32.7)$ & $234(32.2)$ & $44(35.5)$ & 0.476 \\
\hline Metabolic syndrome & $309(36.4)$ & $224(30.9)$ & $85(68.5)$ & $<0.001$ \\
\hline Hypertension & $413(48.6)$ & $342(47.1)$ & $71(57.3)$ & 0.001 \\
\hline Diabetes mellitus & $144(16.9)$ & $95(13.1)$ & $49(39.5)$ & $<0.001$ \\
\hline \multicolumn{5}{|l|}{ Pulmonary Function Test } \\
\hline FVC (\%) & $89.0 \pm 15.5$ & $89.7 \pm 15.5$ & $85.3 \pm 15.3$ & 0.004 \\
\hline $\mathrm{FEV}_{\text {। }}(\%)$ & $76.3 \pm 16.4$ & $76.6 \pm 16.8$ & $74.9 \pm 13.9$ & 0.240 \\
\hline Ratio of $\mathrm{FEV}_{1} / \mathrm{FVC}(\%)$ & $0.6 \pm 0.1$ & $0.6 \pm 0.1$ & $0.6 \pm 0.1$ & 0.002 \\
\hline Systolic blood pressure $(\mathrm{mmHg})$ & $125.0 \pm 16.8$ & $124.5 \pm 16.8$ & $128.4 \pm 16.6$ & 0.017 \\
\hline Diastolic blood pressure $(\mathrm{mmHg})$ & $75.1 \pm 10.3$ & $74.5 \pm 10.3$ & $78.3 \pm 9.7$ & $<0.001$ \\
\hline Total cholesterol (mg/dL) & $190.2 \pm 36.1$ & $190.9 \pm 35.5$ & $186.5 \pm 39.3$ & 0.211 \\
\hline HDL cholesterol (mg/dl) & $45.0 \pm 10.5$ & $45.6 \pm 10.6$ & $41.7 \pm 9.3$ & $<0.001$ \\
\hline Triglyceride (mg/dl) & $146.7 \pm 93.6$ & $139.7 \pm 84.2$ & $187.1 \pm 129.4$ & $<0.001$ \\
\hline AST (IU/L) & $23.1 \pm 8.5$ & $22.8 \pm 8.0$ & $25.0 \pm 10.8$ & $<0.001$ \\
\hline ALT (IU/L) & $21.1 \pm 12.1$ & $19.0 \pm 9.4$ & $33.1 \pm 18.0$ & 0.025 \\
\hline Platelet $\left(\times 10^{9} / \mathrm{L}\right)$ & $246.1 \pm 60.2$ & $246.7 \pm 59.9$ & $242.7 \pm 62.0$ & 0.497 \\
\hline Glucose (mg/dl) & $102.1 \pm 23.6$ & $99.7 \pm 20.7$ & $116.2 \pm 32.7$ & $<0.001$ \\
\hline
\end{tabular}

Note: Data are mean \pm standard error (range) or number (percentage).

Abbreviations: ALT, alanine aminotransferase; ASM, appendicular skeletal muscle mass; AST, aspartate aminotransferase; BMI, body mass index; BW, body weight; COPD, chronic obstructive pulmonary disease; $\mathrm{FEV}_{1}$, forced expiratory volume in I sec; FVC, force vital capacity; HDL, high-density lipoprotein; NAFLD, nonalcoholic fatty liver disease. 


\section{Association Between Sarcopenia and NAFLD in Patients with COPD}

Table 2 shows the association between sarcopenia and NAFLD in multivariable logistic regression analyses. Using the definition of sarcopenia_BMI, sarcopenia was associated with the presence of NAFLD in COPD subjects (odds ratio [OR] 1.74, $95 \%$ confidence interval $[\mathrm{CI}], 1.09-2.77 ; \mathrm{p}=0.020)$. This association was consistently maintained after adjusting for age and sex (model 1; OR 2.69, 95\% CI 1.59-4.55; p < 0.001 ), after additional adjustment for FVC (model 2; OR $2.59,95 \%$ CI 1.53-4.39; $\mathrm{p}<0.001$ ), after additional adjustment for hypertension and diabetes mellitus (model 3; OR 2.19, 95\% CI 1.29-3.72; $\mathrm{p}<0.004)$, and after additional adjustment for metabolic syndrome (model 4; OR 1.95, 95\% CI 1.11-3.46; $\mathrm{p}=0.022$ ). Using the definition of sarcopenia_BW, sarcopenia was also associated with NAFLD based on model 1 (OR 2.99; 95\% CI, 1.82-4.95; p < 0.001), model 2 (OR 2.88; 95\% CI, 1.73-4.81; $<<0.001$ ), model 3 (OR 2.57; 95\% CI, 1.51-4.39; $\mathrm{p}<0.001$ ), and model 4 (OR 2.25; 95\% CI, 1.30-3.92; $\mathrm{p}=0.004$ ). However, using the definition of sarcopenia_height, sarcopenia was not associated with NAFLD based on model 1 (OR 1.15; 95\% CI, 0.77-1.72; $\mathrm{p}=0.500)$, model 2 (OR 1.01; 95\% CI, 0.73-1.65; $\mathrm{p}=0.659$ ), model 3 (OR 1.01; 95\% CI, $0.70-1.70 ; \mathrm{p}=0.692$ ), and model 4 (OR 1.06; 95\% CI, 0.67$1.68 ; \mathrm{p}=0.800)$. No differences in the prevalence of NAFLD were observed between COPD GOLD grades (Figure 2A). When analyzed in two groups (GOLD I and GOLD II-IV), COPD GOLD II-IV patients were tended to have NAFLD compared with COPD GOLD I (Figure 2B).

\section{Association Between Sarcopenia and Fibrotic Burden in NAFLD Subjects}

Figure 3 reveals the association between sarcopenia and fibrotic burden in NAFLD subjects. The proportion of sarcopenia_BMI was higher in patients with a high fibrotic burden from NAFLD (Q3, Q4) than in patients with a low fibrotic burden from NALFD (Q1, Q2) (54.8\% vs 24.2\%, $\mathrm{p}=0.024)$. The proportion of sarcopenia_BW was also higher in subjects with a high fibrotic burden than in subjects with a low fibrotic burden $(51.6 \%$ vs $30.6 \%, \mathrm{p}=0.029)$.

\section{Discussion}

This study demonstrated that sarcopenia is associated with NAFLD in patients with COPD. This association was significant after adjusting for age, sex, FVC, hypertension, diabetes mellitus, and metabolic syndrome. Sarcopenia in COPD was also associated with fibrotic burden in NAFLD patients. The proportion of sarcopenia was higher in patients with a high fibrotic burden from NAFLD than in patients with a low fibrotic burden from NAFLD.

Several studies have demonstrated the relationship between COPD and NAFLD. Low grade inflammation, chronic intermittent hypoxia, and low physical activity are contributing factors to the development of NALFD in patients with COPD. ${ }^{3}$

Viglino et $\mathrm{al}^{3}$ revealed that the prevalence of NAFLD is high in patients with COPD. In that study, the prevalence of NAFLD was based on noninvasive ultrasonography. The prevalence rates of steatosis, NASH, and fibrosis were $41.4 \%$, $36.9 \%$, and $61.3 \%$, respectively, in patients with COPD. Tumor necrosis factor-alpha (TNF- $\alpha$ ) levels were significantly higher in patients with NAFLD than in patients without NAFLD. Liver fibrosis was a significant risk factor for cardiovascular events and death during the 5-year follow-up of the same cohort of COPD patients. ${ }^{8}$ Moon et $\mathrm{al}^{22}$ revealed that the prevalence of NAFLD was $30.2 \%$ in patients with obstructive lung disease based on a previously validated NAFLD liver fat score. $^{23}$ Other studies have revealed an association between impaired lung function and NAFLD. ${ }^{5,22,24}$ In our study, the prevalence of NAFLD was $14.6 \%$ (124/850), and FVC was

Table 2 Association Between Sarcopenia and NAFLD in COPD Patients

\begin{tabular}{|c|c|c|c|c|c|c|}
\hline & $\begin{array}{l}\text { Sarcopenia_BMI } \\
\text { OR }(95 \% \mathrm{CI})\end{array}$ & $P$ value & $\begin{array}{l}\text { Sarcopenia_BW } \\
\text { OR }(95 \% \mathrm{CI})\end{array}$ & $P$ value & $\begin{array}{l}\text { Sarcopenia_Height } \\
\text { OR }(95 \% \mathrm{Cl})\end{array}$ & $P$ value \\
\hline Unadjusted & $1.74(1.09-2.77)$ & 0.020 & $2.44(1.47-4.03)$ & $<0.001$ & I.I6 (0.78-I.72) & 0.476 \\
\hline Multivariable Model I & $2.69(1.59-4.55)$ & $<0.001$ & $2.99(1.82-4.95)$ & $<0.001$ & I.IS (0.77-I.72) & 0.500 \\
\hline Multivariable Model 2 & $2.59(1.53-4.39)$ & $<0.001$ & $2.88(I .73-4.8 I)$ & $<0.001$ & I.0I (0.73-1.65) & 0.659 \\
\hline Multivariable Model 3 & $2.19(1.29-3.72)$ & $<0.004$ & $2.57(1.51-4.39)$ & $<0.001$ & I.0I (0.70-I.70) & 0.692 \\
\hline Multivariable Model 4 & $1.95(1.11-3.46)$ & 0.022 & $2.25(1.30-3.92)$ & 0.004 & I.06 (0.67-I.68) & 0.800 \\
\hline
\end{tabular}

Notes: The multivariable model was not adjusted for ASM and BMI, as these variables were used for calculating definition of sarcopenia. Model I $=$ adjusted for age, gender. Model $2=$ model $1+$ forced vital capacity. Model $3=$ model $2+$ hypertension, diabetes mellitus. Model $4=$ model $3+$ metabolic syndrome

Abbreviations: ASM, appendicular skeletal muscle mass; BMI, body mass index; BW, body weight; $\mathrm{Cl}$, confidence interval; COPD, chronic obstructive pulmonary disease; NAFLD, nonalcoholic fatty liver disease; OR, odds ratio. 

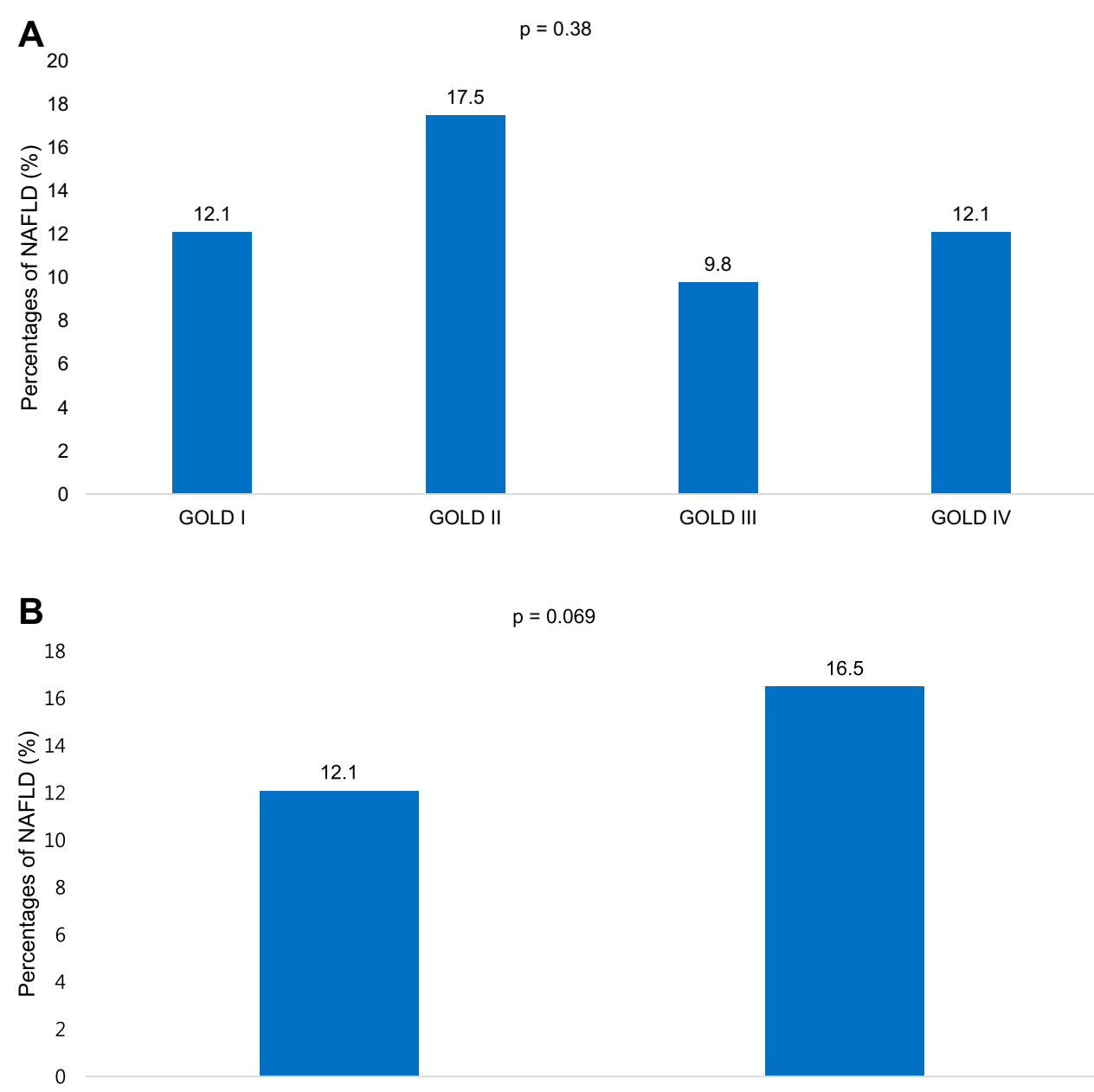

$p=0.069$

GOLD I

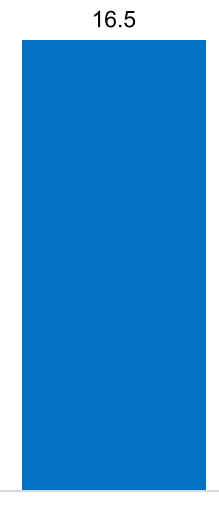

GOLD II-IV

Figure 2 Prevalence of NAFLD in patients with COPD based on the GOLD grade. (A) GOLD I, II, III, and IV; (B) GOLD I, and II-IV.

Abbreviations: COPD, chronic obstructive pulmonary disease; GOLD, global initiative for chronic obstructive lung disease; NAFLD, nonalcoholic fatty liver disease.

lower in NAFLD subjects than in those without NAFLD. The prevalence of NAFLD in our study was lower than that in previous studies performed in COPD patients. ${ }^{3,22}$ The gold standard for diagnosing NAFLD (liver biopsy) was not feasible in such studies, and the definition of NAFLD has varied from study to study, which explains the variation in the prevalence of NALFD between studies.

Skeletal limb muscle dysfunction frequently occurs in patients with COPD. The sarcopenia prevalence rates in COPD patients have been reported to be $15-25 \%$ in previous studies. $^{25-27}$ In our study, the prevalence rates of sarcopenia in all COPD patients were 20.5\% (Sarcopenia_BMI) and 21.3\% (Sarcopenia_BW), respectively. Chronic inflammation, oxidative stress, smoking, hypoxemia, physical inactivity, nutritional imbalance, and the use of systemic corticosteroid are contributing factors to the development of sarcopenia in COPD patients. ${ }^{9,28}$ Sarcopenia has a negative effect on physical activity, exercise tolerance, quality of life, mortality, and metabolism in COPD patients. Muscle atrophy, shift in fiber type, mitochondrial dysfunction, and poor oxidative capacity are morphological and structural changes that occur in limb muscles of patients with stable COPD. ${ }^{29}$ Sarcopenia associated with COPD can be managed with exercise, nutritional support, and medications, such as vitamin D. ${ }^{9}$

Several studies have demonstrated an association between sarcopenia and NAFLD in the general population. Furthermore, sarcopenia is associated with advanced fibrosis, independent of obesity and metabolic components. ${ }^{10-12,30,31}$ A recent study showed that increasing skeletal muscle mass over time can help reduce the development of NAFLD or resolve preexisting NAFLD. ${ }^{32}$ Sarcopenia and NAFLD share common pathophysiological mechanisms, such as insulin resistance, vitamin D deficiency, and chronic low-grade inflammation. $^{13}$ 

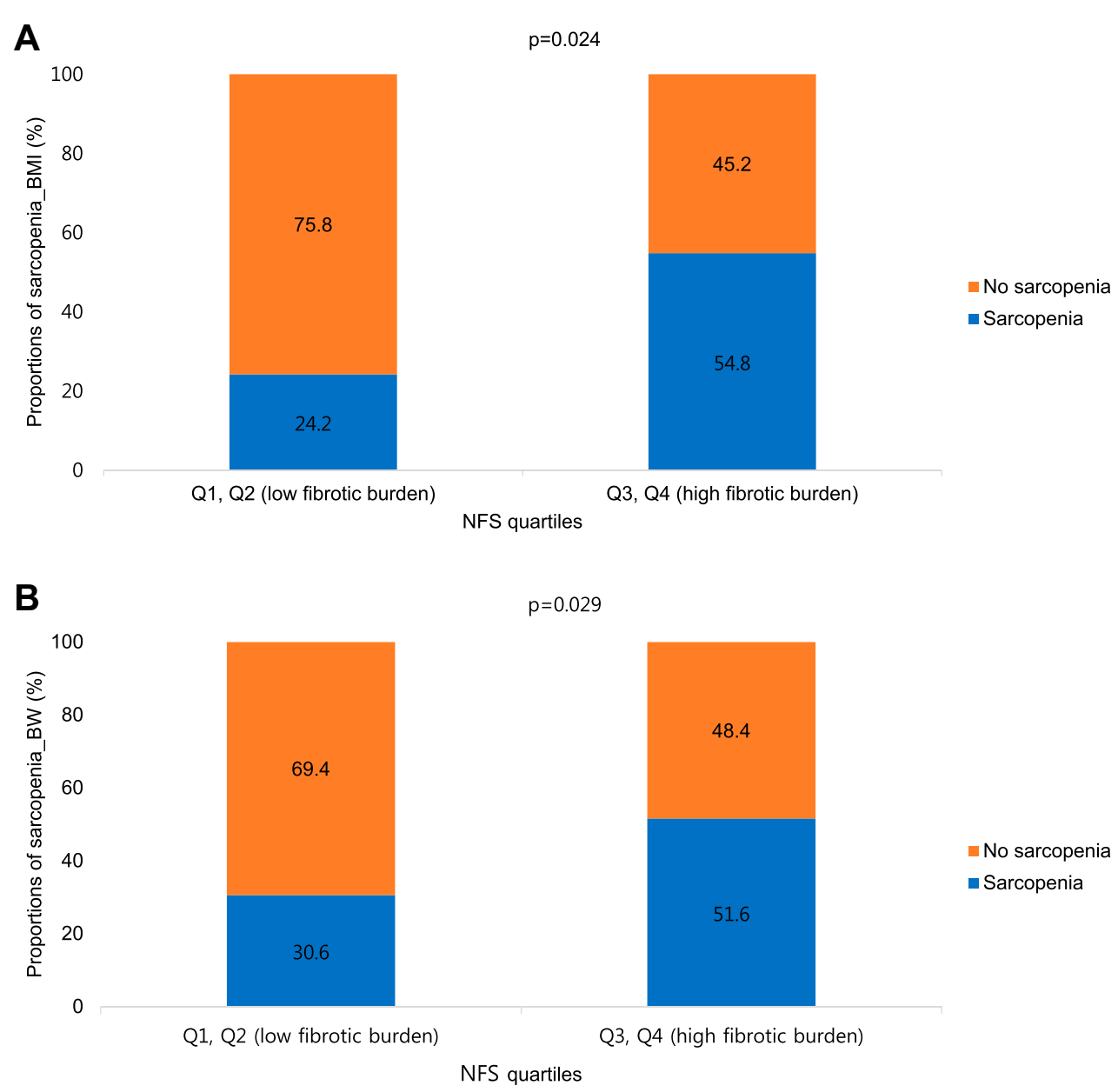

Figure 3 Association between sarcopenia and fibrotic burden in NAFLD patients $(n=124)$ according to the definitions of sarcopenia. (A) Sarcopenia_BMI; (B) sarcopenia_BW.

Abbreviations: BMI, body mass index; BW, body weight; NAFLD, nonalcoholic fatty liver disease; NFS, NAFLD fibrosis score.

Skeletal muscle plays an important role in glucose metabolism. Loss of skeletal muscle mass (sarcopenia) may lead to reduced insulin signaling and action, which is called insulin resistance. Insulin resistance enhances hepatic fat accumulation by increasing free fatty acid delivery to the liver, resulting in NAFLD. ${ }^{33,34}$ Many studies have also demonstrated that a low vitamin D level is associated with an increased risk for sarcopenia and NAFLD. ${ }^{30,35-37}$

Chronic low-grade inflammation is also a common mechanism. Levels of pro-inflammatory mediators, such as interleukin-6 (IL-6) and TNF- $\alpha$, increase in sarcopenia patients. ${ }^{38}$ High serum levels of TNF- $\alpha$ are associated with the development and progression of NAFLD. ${ }^{39}$ TNF- $\alpha$ promotes liver inflammation, lipid accumulation, and apoptosis of liver cells. ${ }^{40,41}$ It is also associated with insulin resistance, which is the main cause of NAFLD. ${ }^{42}$

Lee et $\mathrm{al}^{12}$ reported the relationship between sarcopenia and NAFLD in the general population over 20 years, based on nationwide survey (KNHANES 2008-2011). The study adopted sarcopenia_BW as the definition of sarcopenia. Sarcopenia was associated with NAFLD after adjustment for BMI, diabetes status, triglyceride levels and insulin resistance (OR 1.18; 95\% CI, 1.03-1.34; $\mathrm{p}=$ 0.014 ) in the study. In comparison, our study showed the association between sarcopenia and NAFLD in a COPD population with adjusted OR 2.25, using the definition of sarcopenia_BW. Considering similar research designs for the Korean by two studies, the relationship between sarcopenia and NAFLD is thought to be stronger in COPD patients than in the general population. Future research is needed on why there is a stronger relationship in COPD patients.

To the best of our knowledge, this is the first study demonstrating an association between sarcopenia and NAFLD in COPD patients. The common mechanisms between sarcopenia and NAFLD (insulin resistance, vitamin D deficiency, and chronic low-grade inflammation) are also 
common problems in COPD. First, low physical activity, inflammatory and oxidative stress, abnormal body composition and the steroid treatment contribute to insulin resistance in COPD. ${ }^{43}$ Second, vitamin D deficiency is highly prevalent in COPD patients, and vitamin D supplementation reduces COPD exacerbations in patients with a low vitamin D level. ${ }^{44,45}$ Third, COPD is a chronic inflammatory disease that mainly occurs in the peripheral airways and lung parenchyma, and is characterized by macrophages, neutrophils, and type 1 cytotoxic T cells. ${ }^{46}$ Sarcopenia, NAFLD, and COPD share such common mechanisms, and sarcopenia is associated with NAFLD in patients with COPD after adjusting for several confounding factors.

Several limitations in this study should be discussed. First, although liver biopsy is the gold standard for diagnosing NAFLD and assessing liver fibrosis severity, it was not available in this study. We used the HSI as a validated noninvasive prediction model, and HSI $>36$ was used to define NAFLD. A large cross-sectional study conducted in Korea showed that the HSI has an area under the receiver-operating curve of 0.812 for diagnosing NAFLD. At values $>36.0$, the HSI detected NAFLD with specificity of $93.1 \%$ (95\% CI, 92.0-94.0) and a positive likelihood ratio of $6.505(95 \%$ CI 5.628-7.519). ${ }^{16}$ Thus, a definition of NAFLD based on the HSI can be considered appropriate for the Korean population. We also used the NFS well-validated fibrosis predictive model to assess liver fibrosis severity. ${ }^{17}$ Second, as this study was a cross-sectional design, we could not confirm the definite and dynamic relationship between sarcopenia and NAFLD. More studies are needed to reveal the dynamic association between changes in skeletal muscle mass and the development or progression of NAFLD. Third, using the definition of sarcopenia_height, sarcopenia was not associated with NAFLD in this study. The definition of sarcopenia varies from group to group. More studies are needed using various definitions of sarcopenia, to clarify the relationship between sarcopenia an NAFLD. Fourth, we could not adjust for confounders, such as markers of inflammation or physical activity.

Our study also had several strengths. First, this is the first study demonstrating an association between sarcopenia and NAFLD in COPD patients based on a noninvasive diagnostic tool. Although sarcopenia and NAFLD are common problems in patients with COPD, no study has evaluated the association between sarcopenia and NAFLD in patients with COPD. Future research on other cohort groups is needed to generalize the results of this study. Second, as this was a large-scale population-based study, the results have strong power and reliability.

In conclusion, we found that sarcopenia was associated with NAFLD in COPD patients. This association was significant after adjusting for age, sex, FVC, hypertension, diabetes mellitus, and metabolic syndrome. Sarcopenic COPD was also associated with fibrotic burden in NAFLD patients. In the sarcopenic COPD phenotype, NAFLD should be considered a possible combined comorbidity. Prospective, longitudinal studies are needed to clarify the relationship between sarcopenia and NAFLD in patients with COPD.

\section{Data Sharing Statement}

The datasets generated during the current study are available from the corresponding author on reasonable request.

\section{Author Contributions}

All authors made substantial contributions to conception and design, acquisition of data, or analysis and interpretation of data; took part in drafting the article or revising it critically for important intellectual content; gave final approval of the version to be published; and agree to be accountable for all aspects of the work.

\section{Disclosure}

The authors declare that they have no conflicts of interest.

\section{References}

1. Chalasani N, Younossi Z, Lavine JE, et al. The diagnosis and management of non-alcoholic fatty liver disease: practice guideline by the American Association for the Study of Liver Diseases, American College of Gastroenterology, and the American Gastroenterological Association. Hepatology. 2012;55(6):2005-2023. doi:10.1002/hep.25762

2. Angulo P. Nonalcoholic fatty liver disease. $N$ Engl J Med. 2002;346 (16):1221-1231 doi: 10.1056/NEJMra011775

3. Viglino D, Jullian-Desayes I, Minoves M, et al. Nonalcoholic fatty liver disease in chronic obstructive pulmonary disease. Eur Respir $J$. 2017;49(6). doi:10.1183/13993003.01923-2016

4. Minakata Y, Ueda H, Akamatsu K, et al. High COPD prevalence in patients with liver disease. Intern Med. 2010;49(24):2687-2691. doi:10.2169/internalmedicine.49.3948

5. Jung DH, Shim JY, Lee HR, Moon BS, Park BJ, Lee YJ. Relationship between non-alcoholic fatty liver disease and pulmonary function. Intern Med J. 2012;42(5):541-546 doi: 10.1111/j.1445-5994.2011.02644.x

6. Peng TC, Kao TW, Wu LW, et al. Association between pulmonary function and nonalcoholic fatty liver disease in the NHANES III study. Medicine. 2015;94(21):e907. doi:10.1097/MD.0000000000000907

7. Qin L, Zhang W, Yang Z, et al. Impaired lung function is associated with non-alcoholic fatty liver disease independently of metabolic syndrome features in middle-aged and elderly Chinese. BMC Endocr Disord. 2017;17(1):18. doi:10.1186/s12902-017-0168-4 
8. Viglino D, Plazanet A, Bailly S, et al. Impact of non-alcoholic fatty liver disease on long-term cardiovascular events and death in chronic obstructive pulmonary disease. Sci Rep. 2018;8(1):16559. doi:10.1038/s41598-018-34988-2

9. Kim SH, Shin MJ, Shin YB, Kim KU. Sarcopenia associated with chronic obstructive pulmonary disease. J Bone Metab. 2019;26 (2):65-74 doi: $10.11005 / \mathrm{jbm} .2019 .26 .2 .65$

10. Koo BK, Kim D, Joo SK, et al. Sarcopenia is an independent risk factor for non-alcoholic steatohepatitis and significant fibrosis. J Hepatol. 2017;66(1):123-131. doi:10.1016/j.jhep.2016.08.019

11. Lee YH, Kim SU, Song K, et al. Sarcopenia is associated with significant liver fibrosis independently of obesity and insulin resistance in nonalcoholic fatty liver disease: nationwide surveys (KNHANES 2008-2011). Hepatology. 2016;63(3):776-786. doi:10. 1002/hep. 28376

12. Lee YH, Jung KS, Kim SU, et al. Sarcopaenia is associated with NAFLD independently of obesity and insulin resistance: nationwide surveys (KNHANES 2008-2011). J Hepatol. 2015;63(2):486-493. doi:10.1016/j.jhep.2015.02.051

13. Zhai Y, Xiao Q. The common mechanisms of sarcopenia and NAFLD. Biomed Res Int. 2017;2017:6297651. doi:10.1155/2017/ 6297651

14. Kweon S, Kim Y, Jang MJ, et al. Data resource profile: the Korea National Health and Nutrition Examination Survey (KNHANES). Int J Epidemiol. 2014;43(1):69-77. doi:10.1093/ije/dyt228

15. Kim Y. The Korea National Health and Nutrition Examination Survey (KNHANES): current status and challenges. Epidemiol Health. 36:e2014002. 2014; doi:10.4178/epih/e2014002

16. Lee JH, Kim D, Kim HJ, et al. Hepatic steatosis index: a simple screening tool reflecting nonalcoholic fatty liver disease. Dig Liver Dis. 2010;42(7):503-508. doi:10.1016/j.dld.2009.08.002

17. Angulo P, Hui JM, Marchesini G, et al. The NAFLD fibrosis score: a noninvasive system that identifies liver fibrosis in patients with NAFLD. Hepatology. 2007;45(4):846-854. doi:10.1002/hep.21496

18. Grundy SM, Cleeman JI, Daniels SR, et al. Diagnosis and management of the metabolic syndrome: an American Heart Association/ National Heart, Lung, and Blood Institute Scientific Statement Circulation. 2005;112(17):2735-2752. doi:10.1161/CIRCULATION AHA.105.169404

19. Studenski SA, Peters KW, Alley DE, et al. The FNIH sarcopenia project: rationale, study description, conference recommendations, and final estimates. J Gerontology: Series A. 2014;69(5):547-558. doi:10.1093/gerona/glu010

20. Lee YH, Kim JE, Roh YH, et al. The combination of vitamin $\mathrm{D}$ deficiency and mild to moderate chronic kidney disease is associated with low bone mineral density and deteriorated femoral microarchitecture: results from the KNHANES 2008-2011. J Clin Endocrinol Metab. 2014;99(10):3879-3888. doi:10.1210/jc.20133764

21. Chen LK, Liu LK, Woo J, et al. Sarcopenia in Asia: consensus report of the Asian Working Group for Sarcopenia. J Am Med Dir Assoc. 2014;15(2):95-101. doi:10.1016/j.jamda.2013.11.025

22. Moon SW, Kim SY, Jung JY, et al. Relationship between obstructive lung disease and non-alcoholic fatty liver disease in the Korean population: Korea National Health and Nutrition Examination Survey, 2007-2010. Int J Chron Obstruct Pulmon Dis. 2018;13:2603-2611. doi:10.2147/COPD.S166902

23. Kotronen A, Peltonen M, Hakkarainen A, et al. Prediction of non-alcoholic fatty liver disease and liver fat using metabolic and genetic factors. Gastroenterology. 2009;137(3):865-872. doi:10.10 53/j.gastro.2009.06.005

24. Song JU, Jang Y, Lim SY, et al. Decreased lung function is associated with risk of developing non-alcoholic fatty liver disease: a longitudinal cohort study. PLoS One. 2019;14(1):e0208736. doi:10. 1371/journal.pone. 0208736
25. Byun MK, Cho EN, Chang J, Ahn CM, Kim HJ. Sarcopenia correlates with systemic inflammation in COPD. Int $J$ Chron Obstruct Pulmon Dis. 2017;12:669-675. doi:10.2147/COPD.S130790

26. Wagner PD. Possible mechanisms underlying the development of cachexia in COPD. Eur Respir J. 2008;31(3):492-501 doi: 10.1183/ 09031936.00074807

27. Jones SE, Maddocks M, Kon SS, et al. Sarcopenia in COPD: prevalence, clinical correlates and response to pulmonary rehabilitation. Thorax. 2015;70(3):213-218. doi:10.1136/thoraxjnl-2014-206440

28. Benz E, Trajanoska K, Lahousse L, et al. Sarcopenia in COPD: a systematic review and meta-analysis. Eur Respir Rev. 2019;28:154. doi:10.1183/16000617.0049-2019

29. Maltais F, Decramer M, Casaburi R, et al. An official American Thoracic Society/European Respiratory Society statement: update on limb muscle dysfunction in chronic obstructive pulmonary disease. Am J Respir Crit Care Med. 2014;189(9):e15-62. doi:10. 1164/rccm.201402-0373ST

30. Hong HC, Hwang SY, Choi HY, et al. Relationship between sarcopenia and nonalcoholic fatty liver disease: the Korean Sarcopenic Obesity Study. Hepatology. 2014;59(5):1772-1778. doi:10.1002/ hep. 26716

31. Wijarnpreecha K, Panjawatanan $\mathrm{P}$, Thongprayoon $\mathrm{C}$, Jaruvongvanich V, Ungprasert P. Sarcopenia and risk of nonalcoholic fatty liver disease: a meta-analysis. Saudi J Gastroenterol. 2018;24 (1):12-17 doi: 10.4103/sjg.SJG_237_17

32. Kim G, Lee SE, Lee YB, et al. Relationship between relative skeletal muscle mass and nonalcoholic fatty liver disease: a 7-year longitudinal study. Hepatology. 2018;68(5):1755-1768. doi:10.1002/ hep.30049

33. Pacifico L, Perla FM, Chiesa C. Sarcopenia and nonalcoholic fatty liver disease: a causal relationship. Hepatobiliary Surg Nutr. 2019;8 (2):144-147 doi: 10.21037/hbsn.2018.11.11

34. Utzschneider KM, Kahn SE. Review: the role of insulin resistance in nonalcoholic fatty liver disease. J Clin Endocrinol Metab. 2006;91 (12):4753-4761 doi: 10.1210/jc.2006-0587

35. Seo JA, Cho H, Eun CR, et al. Association between visceral obesity and sarcopenia and vitamin D deficiency in older Koreans: the Ansan Geriatric Study. J Am Geriatr Soc. 2012;60(4):700-706. doi:10.1111/ j.1532-5415.2012.03887.x

36. Scott D, Blizzard L, Fell J, Ding C, Winzenberg T, Jones G. A prospective study of the associations between 25-hydroxyvitamin D, sarcopenia progression and physical activity in older adults. Clin Endocrinol (Oxf). 2010;73(5):581-587 doi: 10.1111/ j.1365-2265.2010.03858.x

37. Barchetta I, Angelico F, Del Ben M, et al. Strong association between non alcoholic fatty liver disease (NAFLD) and low $25(\mathrm{OH})$ vitamin D levels in an adult population with normal serum liver enzymes. BMC Med. 2011;9:85. doi:10.1186/1741-7015-9-85

38. Beyer I, Mets T, Bautmans I. Chronic low-grade inflammation and age-related sarcopenia. Curr Opin Clin Nutr Metab Care. 2012;15 (1):12-22 doi: 10.1097/MCO.0b013e32834dd297

39. Seo YY, Cho YK, Bae JC, et al. Tumor necrosis factor- $\alpha$ as a predictor for the development of nonalcoholic fatty liver disease: a 4-year follow-up study. Endocrinol Metabol. 2013;28(1):41-45. doi:10.3803/EnM.2013.28.1.41

40. Wang K. Molecular mechanisms of hepatic apoptosis. Cell Death Dis. 5:e996. 2014;doi:10.1038/cddis.2013.499

41. Ma KL, Ruan XZ, Powis SH, Chen Y, Moorhead JF, Varghese Z. Inflammatory stress exacerbates lipid accumulation in hepatic cells and fatty livers of apolipoprotein E knockout mice. Hepatology. 2008;48(3):770-781 doi: 10.1002/hep.22423

42. Hotamisligil GS, Shargill NS, Spiegelman BM. Adipose expression of tumor necrosis factor-alpha: direct role in obesity-linked insulin resistance. Science. 1993;259(5091):87-91 doi: 10.1126/science. 7678183 
43. Machado FVC, Pitta F, Hernandes NA, Bertolini GL. Physiopathological relationship between chronic obstructive pulmonary disease and insulin resistance. Endocrine. 2018;61(1):17-22 doi: 10.1007/s12020-018-1554-z

44. Janssens W, Mathieu C, Boonen S, Decramer M. Vitamin D deficiency and chronic obstructive pulmonary disease: a vicious circle. Vitam Horm. 2011;86:379-399.
45. Jolliffe DA, Greenberg L, Hooper RL, et al. Vitamin D to prevent exacerbations of COPD: systematic review and meta-analysis of individual participant data from randomised controlled trials. Thorax. 2019;74(4):337-345. doi:10.1136/thoraxjnl-2018-212092

46. Barnes PJ. Immunology of asthma and chronic obstructive pulmonary disease. Nat Rev Immunol. 2008;8(3):183-192 doi: 10.1038/ nri2254

\section{Publish your work in this journal}

The International Journal of COPD is an international, peer-reviewed journal of therapeutics and pharmacology focusing on concise rapid reporting of clinical studies and reviews in COPD. Special focus is given to the pathophysiological processes underlying the disease, intervention programs, patient focused education, and self management protocols. This journal is indexed on PubMed Central, MedLine and CAS. The manuscript management system is completely online and includes a very quick and fair peer-review system, which is all easy to use. Visit http://www.dovepress.com/testimonials.php to read real quotes from published authors. 